\title{
Norois
}

Environnement, aménagement, société

\section{Une carte, trois communes et deux États}

Conflits pour l'appropriation institutionnelle de pâturages alpins frontaliers

\section{Lucie Bargel}

\section{Q OpenEdition}

1 Journals

Édition électronique

URL : https://journals.openedition.org/norois/5884

DOI : $10.4000 /$ norois.5884

ISBN : 978-2-7535-5227-2

ISSN : $1760-8546$

Éditeur

Presses universitaires de Rennes

Édition imprimée

Date de publication : 17 octobre 2016

Pagination : 85-95

ISBN : 978-2-7535-5222-7

ISSN : 0029-182X

\section{Référence électronique}

Lucie Bargel, "Une carte, trois communes et deux États », Norois [En ligne], 238-239 | 2016, mis en ligne le 17 octobre 2018, consulté le 13 janvier 2022. URL : http://journals.openedition.org/norois/ 5884 ; DOI : https://doi.org/10.4000/norois.5884 


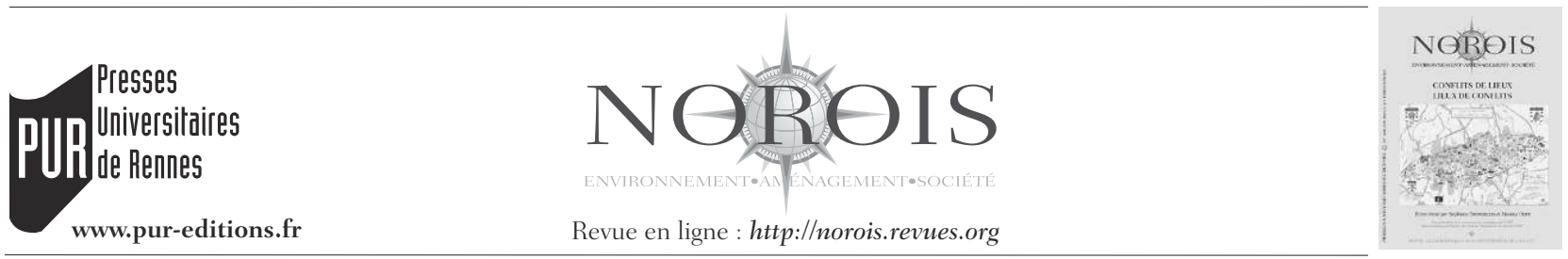

\title{
Une carte, trois communes et deux États Conflits pour l'appropriation institutionnelle de pâturages alpins frontaliers
}

\author{
One Map, Three Villages and Two States \\ Conflicts over the Institutional Appropriation of Alpine Pastures in Border Areas
}

\author{
Lucie BARGEL
}

Université Côte-d'Azur, ERMES et CESSP - Avenue Trotabas, 06050 NICE cedex 01. (bargel@unice.fr)

\begin{abstract}
Résumé : Cet article se situe dans la période de retrait de l'administration italienne et d'installation de son homologue française après le déplacement de la frontière des Alpes du Sud par le traité de paix de 1947. À travers un désaccord relatif à l'usage de pâturages, il pose la question du degré de prise de l'État sur ces territoires de montagne et étudie la saisie institutionnelle de ce lieu, en soulignant la diversité des acteurs engagés dans ce conflit territorial et la multiplicité de rapports au territoire et de formulation d'intérêts « nationaux » qui en découle.
\end{abstract}

\begin{abstract}
This article deals with the period of withdrawal of the Italian administration and of installation of its French counterpart after the moving of the Southern Alps border by the 1947 peace treaty. Through a disagreement on the use of pastures, it addresses the degree of settlement of the state on these relatively isolated highlands studies and considers the institutional capture of this place, highlighting the diversity of actors involved in this territorial dispute and the resulting manifold relationships to the territory and definitions of "national" interests.
\end{abstract}

Mots clés : frontière - nationalisme - administration - local - conflit - France - Italie

Keywords: border - nationalism - administration - local - conflict - France - Italy

Avec quels outils appréhende-t-on un territoire? Depuis les années 1970, la géographie n'est plus la discipline née « à l'ombre de l'armée » et longtemps «impliquée dans la production des discours de légitimation d'une action sur l'espace, au service d'un pouvoir politique dominant, que ce soit par des cartes ou des textes »(Dumont, 2011). En particulier, sa tendance critique insiste depuis une vingtaine d'années sur le caractère socialement et politiquement construit de l'outil « carte » (Wood, 1992 ; 2010). Elle rencontre ainsi les historiens de la cartographie (Harley, 2001) : Kain et Baigent (1992) montrent combien la carte cadastrale, instaurée par l'autorité politique à partir du XVI siècle en Europe, constitue un point d'appui pour toute une série de ses «monopoles» (Weber, 1921) : politique fiscale, 
patrimoniale, etc. Tous insistent sur l'incorporation des intérêts étatiques dans les outils cartographiques.

Plus encore, Amilhat-Szary et Giraut (2015) rappellent que cartes et frontières ont été inventées ensemble et qu'elles continuent d'être consubstantielles. Bourdon le montre à propos de l'appropriation des Alpes : "Nous cherchions les Alpes, nous avons trouvé l'État» (2011, p. 523). Ce n'est en effet qu'à partir de la Renaissance, lorsqu'une autorité politique s'affirme sur un territoire qui doit être connu, contrôlé et fini qu’apparaissent des représentations cartographiques des montagnes. Jusqu'alors, elles n'étaient appréhendées que par l'expérience sensible du voyage, comme autant d'étapes à traverser. À l'époque contemporaine, Radcliffe (2001) souligne que la constitution d'une discipline géographique au Salvador est inséparable des conflits de frontières qui opposent l'État naissant à son voisin péruvien.

Cet article se situe dans la continuité de ces analyses des cartes comme outils de gouvernement, tout en insistant particulièrement sur la dimension concurrentielle de la saisie institutionnelle du territoire. À travers un conflit consécutif au déplacement de la frontière italo-française des Alpes en 1947 (figure 1), il examine les outils que déploient les deux États sur ce territoire disputé, et souligne ainsi combien le travail administratif est toujours imprégné de considérations nationalistes. Mais nous allons également voir que les États centraux ne sont pas les seuls acteurs politiques en concurrence sur ces zones montagnardes. D'abord, les usages locaux du territoire ne sont pas entièrement maîtrisés par le monopole étatique. Ensuite, acteurs locaux, nationaux, et finalement internationaux, défendent des réponses diverses et concurrentes à la question de savoir si les bergers de Realdo, restés italiens, peuvent conserver l'usage de leurs anciens pâturages, devenus français. Précisons dès maintenant que ces pâturages constituent un prétexte pour explorer les manières dont différents acteurs politiques et institutionnels se saisissent de ce conflit local, mais que nous ne nous intéresserons pas à la vie pastorale en soi.

Cet article s'appuie sur le traitement d'une série d'archives publiques : principalement, les archives diplomatiques italiennes et françaises concernant d'une part la conférence de paix de 1947 et d'autre

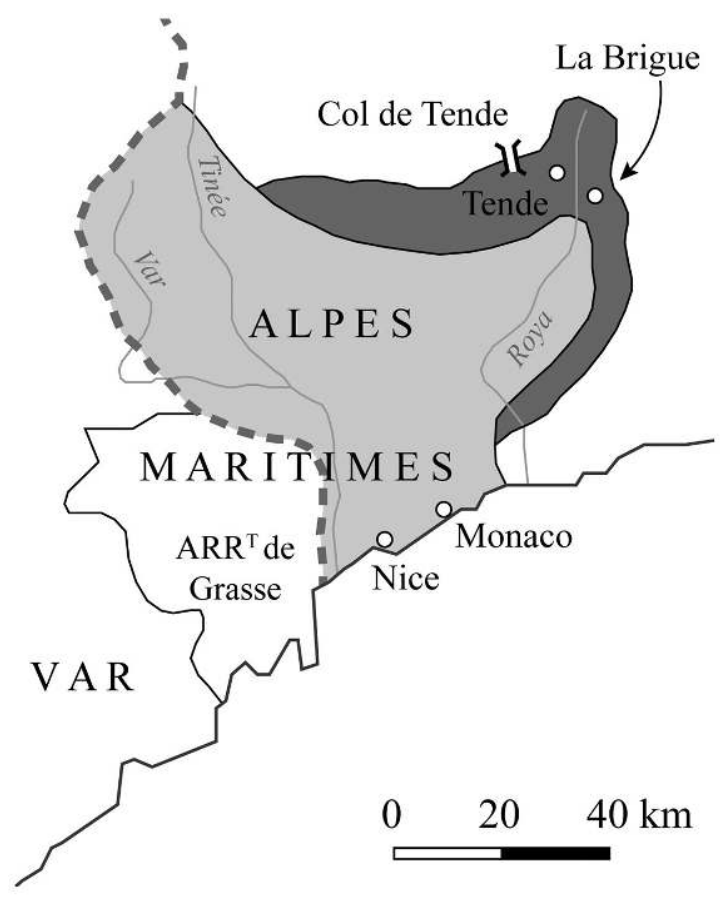

$=-\mathbf{m}$ Frontière de 1790

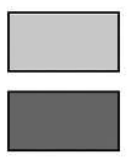

Acquisition de 1860

Acquisition de 1947

\section{Réseau hydrographique}

Figure 1 : Modifications de la frontière sud-est de la France (17901947) (Source : Atlas historique de la France contemporaine : 18001965, Paris, A. Colin 1966)

Alterations to France's southeastern border (1790-1947)

part les relations entre les deux États des années 1930 aux années $1960^{1}$; les archives des cabinets des préfets des Alpes-Maritimes, d'Imperia et de Torino, ces dernières comportant en particulier les archives du président de la Commissione esperti civili per le questioni derivanti dal nuovo confine occidentale $^{2}$ (par la suite CEC), qui n'ont pas d'équivalent côté français ${ }^{3}$; et de façon secondaire, les archives militaires françaises et un recueil d'histoires orales ${ }^{4}$.

1. En Italie, en particulier : Archivio diplomatico, Serie Affari Politici 19311945; Affari Politici 1946-1950; Affari Politici 1951-1957; Gabinetto 1944-1958; DGAP Ufficio I Versamento I 1945-1960 (45 cartons). En France, en particulier : Archives diplomatiques, Série Z Europe 19441970, Italie (60 cartons).

2. Archivio di Stato di Torino, Sezioni Riunite. Gabinetto Prefettura Torino, versamento 1996, buste B107 a B130-2 (25 cartons).

3. Le plus approchant se trouve aux Archives départementales des AlpesMaritimes (par la suite AD06), Cabinet du préfet, série 378W frontière franco-italienne (14 cartons).

4. Enquêtes d'histoires orales dans les vallées de la Roya et de la Bévéra, commanditaires MSH de Nice et AD06. Fonds MMSH, 2005-2009. 
À travers la présentation des différentes étapes du conflit autour de ces pâturages, qui dure jusqu'en 1953, nous montrerons un double processus à l'œuvre : en même temps que l'échelon institutionnel concerné augmente en superficie et en autorité juridique, le degré de technicité des arguments et des instruments de saisie du territoire s'intensifie également. Autrement dit, plus les tentatives de résolution de ce conflit s'éloignent de l'échelon local, et plus elles s'écartent également des logiques politiques qui en sont au principe.

\section{DES APPARTENANCES NATIONALES LOCALEMENT INSTABLES}

Les pâturages disputés présentent deux spécificités qui donnent de l'intensité à ce conflit. D’abord, ils sont situés en montagne, qui reste longtemps peu fréquentée : les militaires sont jusqu'alors les seuls acteurs étatiques à parcourir ces zones, en lien avec les alpinistes dont l'activité se développe avant-guerre (Hoibian, 2008). Ensuite, ils appartiennent à la partie de l'ancien comté de Nice qui, en 1860, n'a pas été rattachée à la France mais est restée au Piémont italien. Cette zone devient française en 1947 : leur appartenance nationale est donc bien moins stabilisée que l'essentiel du territoire d'Europe de l'Ouest de l'époque.

\section{Les effets administratifs du déplacement d'une frontière}

Centrée sur la maîtrise des cols et des crêtes de montagnes, la nouvelle ligne frontière issue du traité de paix ne suit pas les contours des communes: dans les Alpes, elle en traverse dix-huit, dont une partie des biens communaux (forêts, pâturages, etc.) se retrouve alors de l'autre côté de la frontière par rapport au village. Trois d'entre elles perdent également des hameaux et des habitants. Dans ce processus, la commune italienne de Briga Marittima (2026 habitants en 1936) cesse d'exister et est divisée entre France, Piémont et Ligurie : son bourg et un hameau composent la nouvelle commune française de la Brigue (1 415 habitants en 1946), tandis que trois de ses hameaux (300 habitants) forment la nouvelle commune italienne de Briga Alta, appartenant au Piémont. Le dernier hameau, Realdo (350 habitants, 1007 m d'altitude) est rat- taché à Triora (1 757 habitants en 1936), commune ligure dont il est séparé par un sommet et plus de $10 \mathrm{~km}$ de chemin muletier. La nouvelle frontière réduit les terres auxquelles ont accès les Réaldais : comme l'écrit le maire de Triora, « outre les bergers, 27 familles, Realdo compte 80 autres familles qui aujourd'hui se trouvent étranglées sur un territoire rocheux sans campagne [terrains cultivables] ${ }^{5}$. Pour les bergers, alors qu'ils bénéficiaient auparavant d'un droit d'usage sur des pâturages voisins, ces derniers appartiennent désormais à une autre commune, qui entend les louer, et à un autre État, ce qui suppose de soumettre les bergers et leurs troupeaux à des contrôles douaniers et vétérinaires et au versement de taxes et de cautions. Ils prétendent pourtant conserver leur usage de ces pâturages, ce qui déclenche le conflit étudié.

La répartition des biens des dix-huit communes traversées par la nouvelle frontière constitue un dossier épineux de la mise en œuvre du traité de paix. D'un côté, le paragraphe 1 de l'annexe XIV du traité de paix (« dispositions économiques et financières relatives aux territoires cédés ») prévoit que les biens étatiques ou paraétatiques de l'Italie, y compris ceux des collectivités locales, passent sans compensation à l'État successeur ${ }^{6}$. De l'autre, le paragraphe 18 de cette même annexe indique que «les États successeurs et l'Italie concluront des accords répartissant d'une manière juste et équitable les biens de toute collectivité publique locale existante, dont le territoire se trouve divisé par une frontière établie en vertu du présent Traité, et assurant le maintien de ceux des services communaux nécessaires aux habitants qui ne sont pas expressément visés par d'autres dispositions du Traité ». Les bergers de Realdo et leurs soutiens s'appuient sur ce paragraphe.

\section{Un conflit ancré dans l'histoire du « rattachement »}

La nouvelle commune de la Brigue, désormais propriétaire des pâturages de Realdo, est d'abord administrée par une "délégation spéciale » nommée par le préfet des Alpes-Maritimes. Les élections municipales ont lieu en juillet 1949 et Aimable

5. Lettre du 14 août 1951. CEC, B109.

6. On peut supposer que l'usage de la notion de biens paraétatiques sert à inclure les enti pubblici créés en Italie à partir du début du $\mathrm{xx}^{\mathrm{e}}$ siècle, dépendant davantage du pouvoir politique que de la bureaucratie administrative, et multipliés par le régime fasciste (Dormagen, 2008). 
Gastaud, déjà membre de la délégation, est élu maire. En octobre 1951, il devient également conseiller général du canton de Tende, nouvellement créé, qui regroupe les territoires anciennement italiens. Aimable Gastaud est le principal opposant local aux bergers de Realdo et à leurs représentants, et il bénéficie de relations privilégiées avec la préfecture des Alpes-Maritimes ainsi qu'avec les réseaux médecinistes ${ }^{7}$ qui s'installent à ce moment-là dans le département.

Aimable Gastaud est en effet une figure centrale du processus de revendication puis d'appropriation de ces territoires par la France ${ }^{8}$. Depuis l'entredeux-guerres, il est concierge dans un grand hôtel niçois. Comme lui, de nombreux « originaires » de Briga Marittima travaillent sur le littoral français, dont l'activité touristique d'hiver fournit des emplois temporaires aux agriculteurs du haut pays contraints à l'inactivité par le gel (Bargel, 2016). Ces « originaires » composent les rangs du Comité de rattachement de Tende et Brigue à la France, créé à la Libération et qui s'impose progressivement comme l'intermédiaire entre les pouvoirs politiques et militaires français et les populations concernées. Après 1947, en tant que membre de la délégation spéciale puis maire, Aimable Gastaud reste un interlocuteur important des représentants de l'État français, à qui il écrit de nombreuses lettres pour obtenir des réparations pour la vallée, dont les routes et les ponts ont été détruits lors du retrait des troupes allemandes, et la résolution des multiples questions posées par le transfert de souveraineté (retraites non versées, épargne bloquée dans les banques italiennes, obtention de la nationalité française sous conditions, etc.).

Ces échanges de lettres, et plus largement les archives françaises et italiennes consultées, révèlent la conception de l'attachement national de ces populations qui oriente l'action des pouvoirs politiques. La volonté des populations constitue en effet l'argument principal des revendications italiennes comme françaises sur ces territoires : en cette fin de seconde guerre mondiale, on ne saurait parler d'annexion et c'est le «droit des peuples à disposer d'eux-mêmes » qui doit prévaloir. D'abord, l'atta-

7. Jean Médecin, avocat, est maire de Nice de 1928 jusqu’à sa mort en 1965. Il est également député et sénateur de 1932 à 1962, et président du Conseil général de 1951 à 1961. Ces réseaux médecinistes n’ont malheureusement pas fait l'objet de travaux scientifiques.

8. Pour plus de détails sur sa biographie, cf. celle établie par son petit-fils, maire de la Brigue de 2008 à 2014 (Couttenier et Gastaud, 2007) chement national de ces populations frontalières ne s'exprime pas directement mais passe par leur patronage par des acteurs politiques et religieux locaux. Côté français, Aimable Gastaud tient ce rôle à la mairie de la Brigue. À Realdo, Don Guido Pastor, l'ancien curé italien de Briga Marittima réaffecté à ce hameau, soutient les bergers et les représente auprès des autorités. Nilla Gismondi, présidente du Comitato per l'italianità della valle Roja, et épouse du député-maire de San Remo, s'impose également comme une véritable intermédiaire entre les bergers réaldais et les autorités italiennes. Par exemple, en octobre 1952, le représentant de l'administration italienne lui écrit pour savoir « quels sont en définitive les biens dont le hameau de Realdo aspire à être propriétaire ». Elle lui répond en experte : «Le maire de Triora m'a remis une note [...] qui est en substance conforme à celle des représentants des bergers, s'en distinguant seulement dans la nomenclature. Il me semble cependant plus opportun de se servir de la note des bergers en ceci qu'elle est plus explicative et qu'y sont utilisés, pour indiquer les localités, les termes déjà adoptés dans le compterendu de la réunion du 4 mai $1949^{9}$. »

Cette lettre montre que N. Gismondi s'emploie à acclimater les catégories administratives (« la nomenclature ») et à les faire correspondre aux revendications des bergers. Comme l'indique le nom du comité qu'elle préside (" pour l'italianité de la vallée de la Roya »), tout en s'employant à influencer les modalités d'application du traité de paix, elle en combat le principe de déplacement de la frontière. Comme elle, comme le curé Don Pastor ou encore comme Aimable Gastaud, les acteurs locaux, d'inégal pouvoir institutionnel, impliqués dans le conflit relatif aux pâturages de Realdo sont tous également engagés dans l'histoire du rattachement, et continuent à défendre l'appartenance de la vallée de la Roya à l'un des deux États. Pour tous ces acteurs politiques en effet, la position de la nouvelle frontière semble encore susceptible de se déplacer, d'un côté comme de l'autre, et les prises de positions politiques locales sont indexées sur ce clivage fondamental : France ou Italie.

Ensuite, dans les archives comme dans les témoignages d'histoire orale, il semble communément admis que l'attachement national des populations

9. CEC, B109. 
est directement dépendant du traitement que leur réserve chacun des États, sans que cela n'entre en contradiction avec des déclarations d'amour patriotique. De ce fait, le caractère « filo-français ${ }^{10}$ » ou «filo-italien » des populations n'est jamais acquis et fait l'objet d'évaluations mouvantes selon les circonstances. Durant l'été 1950 par exemple, les notes des renseignements généraux français interprètent l'attitude des bergers réaldais comme une manifestation d'hostilité à l'égard de la France, et non simplement à l'égard de la règlementation douanière, et l'attribuent au fait qu'ils sont « activement travaillés par la propagande anti française » à laquelle se livreraient en particulier Louis Lanteri, le représentant des bergers, et Don Guido Pastor. Cette note traduit une tendance plus générale à sur-interpréter en termes nationalistes les comportements des populations locales. On retrouve cette même logique dans les archives italiennes. En décembre 1949, le cabinet du ministère de l'Intérieur avertit le préfet d'Imperia de «l'intense propagande » menée par les « éléments francophiles » auprès de la population de Realdo dans laquelle persiste "un vif mécontentement » face à cette situation non réglée. En conséquence, la population continue de migrer vers le littoral ligure ou, "même, dans la France voisine $^{11} »$. Il invite donc le préfet à montrer à ces populations qu'à travers lui l'État italien s'emploie à faire aboutir leurs demandes. L'attention accordée par les autorités politico-administratives à ces villages montagnards est, on le voit, inséparable de leur situation frontalière et de la crainte, de part et d'autre de la frontière, que de nouvelles revendications territoriales soient formulées par leur voisine et susceptibles de rencontrer un soutien populaire.

\section{L'ÉCHEC DES SOLUTIONS ADMINISTRATIVES FACE AUX ACTEURS POLITIQUES LOCAUX}

Cette prégnance des logiques nationalistes n'empêche pas que les États eux-mêmes, et leurs appareils bureaucratiques, n'aient qu'une prise limitée sur ces territoires. Certes, l'appropriation étatique de ce territoire montagnard ne débute pas en 1947; mais

10. Les documents présents dans les archives françaises reprennent une traduction et une orthographe littérales du terme italien «filofrancese », plutôt que le terme «francophile».

11. CEC, B1 14. Toutes les traductions de l'italien sont celles de l'auteure. elle reste alors largement imparfaite. En témoignent les difficultés de la commission militaire chargée de placer les nouvelles bornes frontières alors que « texte et trait rouge de la carte du traité ont été établis sur des documents cartographiques inexacts et périmés ${ }^{12} »$. Pour y pallier, le lieutenant français stationné dans la zone avait recommandé que «de vieux bergers ou de vieux chasseurs de Tende et la Brigue soient adjoints, comme guides, aux experts militaires français et italiens ${ }^{13}{ }$. Pour lui, donc, les acteurs civils locaux connaissent mieux la montagne que les fonctionnaires étatiques. L'inachèvement de l'appropriation étatique du territoire se marque encore dans l'absence de consensus autour d'instruments d'action publique pour identifier et évaluer les pâturages concernés par le conflit ${ }^{14}$.

\section{L'absence de consensus sur les instruments}

Parallèlement aux mobilisations locales, les acteurs administratifs s'emploient à appliquer les dispositions du traité. En novembre 1948 commencent les rencontres entre les deux présidents, français et italiens, des commissions des experts civils pour la nouvelle frontière. Le président italien, dont nous mobilisons les archives, est l'avocat Adolfo De Dominicis, Prefetto Ispettore generale; il représente le ministère de l'Intérieur et reste en poste jusqu'à la disparition de cette commission en juin 1956. Côté français, Roger Wuillaume est pour sa part Inspecteur général de l'administration au ministère de l'Intérieur; il sera remplacé en mai 1952. Chacun préside une commission qui regroupe des représentants des différentes administrations nationales concernées par le déplacement de la frontière ${ }^{15}$.

12. Rapport confidentiel sur le travail effectué par la sous-commission 6 bis en 1947, 29 novembre 1947. Archives militaires françaises (par la suite AMF), Série Q Secrétariat général de la défense nationale 1944-1978, 4Q 151 .

13. AMF, 4Q143.

14. Cela peut sembler paradoxal dans la mesure où les deux États partagent par ailleurs une même démarche de codification nationale des droits agricoles locaux. En Italie, le Commissariato per la liquidazione degli usi civici, créé en 1927, reprend après-guerre sa mission « d'écriture et de conservation des usages concernant des terres communales de nature sylvo-pastorale », toujours en vigueur. En France, les chambres d'agriculture créées en 1924 doivent également " grouper, coordonner, codifier les coutumes et usages locaux à caractère agricole »; le premier Code rural est adopté en 1955 (Assier-Andrieu, 1990).

15. La commission italienne regroupe ainsi un conseiller d'État qui représente la présidence du Conseil des ministres, un inspecteur général des douanes pour le ministère des Finances, deux inspecteurs généraux 
Dès leur première rencontre, les deux présidents conviennent d'une solution qu'ils « recommandent à leurs Gouvernements » pour l'application du paragraphe 18 de l'annexe XIV du Traité de paix ${ }^{16}$. Selon eux, les communes qui ont perdu des zones de territoire dépourvues de population devraient en conserver la propriété : elles détiendraient ainsi des biens privés de l'autre côté de la frontière. Pour les communes ayant perdu des habitants, il faudrait procéder à la séparation patrimoniale et financière en fonction des chiffres de la population vivant désormais en zone française ou italienne. De novembre 1948 à septembre 1949, cinq autres rencontres entre les présidents des deux commissions ont lieu afin de fixer les pourcentages de répartition des biens communaux. À leur issue, seuls les biens de l'ancienne commune de Briga Marittima continuent de faire l'objet d'un désaccord entre les estimations italienne et française de leur valeur. Ce désaccord tient très largement à un écart dans les instruments de saisie du territoire utilisés : la zone n'est pas encore intégrée au cadastre français et les catégories cadastrales italiennes et françaises sont différentes.

"Il n'a pas été possible au président de la commission française de demander au seul ingénieur du cadastre une étude similaire à celle établie par l'ingénieur Gerbino. En effet, les autorités françaises ne sont pas encore officiellement en possession des archives cadastrales italiennes qui devaient leur être remises, et, de plus, il n'était pas possible aux services compétents de vérifier l'exactitude des classifications de chaque parcelle entre les diverses catégories prévues au cadastre italien. C'est donc à l'aide des divers fonctionnaires du cadastre, des eauxet-forêts et des services agricoles, et à la lumière tant d'observations faites sur place que des indications fournies par les bergers et par les municipalités de la Brigue et des communes voisines, que le président de la commission française a pu procéder à une étude précise et objective de l'évaluation des biens communaux de l'ex-commune de Brigue-Maritime [Briga Marittima] ${ }^{17}$. »

techniques qui représentent le ministère des Travaux publics, un Inspecteur général ANAS (routes d'État) pour cette section du ministère des Travaux publics, un inspecteur agraire et un inspecteur forestier représentant le ministère de l'Agriculture et des forêts, et un inspecteur du ministère des Transports. Decreto del Presidente del Consiglio dei ministri, 13 octobre 1948.

16. CEC, B 117.

17. Lettre de Wuillaume à De Dominicis, 10 mars 1949. CEC, B109.
Les acteurs politiques locaux, bergers et élus, sont donc intégrés au processus administratif parce qu'ils sont les seuls à disposer d'une connaissance suffisante du territoire.

\section{Le débordement de la saisie administrative de l'espace}

À l'été 1950, dans la commune voisine d'Ormea, les maires des trois communes concernées par le partage des biens brigasques, Briga Alta, Triora et la Brigue, signent des accords «provisoires » prévoyant que la commune de la Brigue octroie aux bergers de Realdo l'usage de trois pâturages situés sur son territoire en contrepartie des mêmes taxes communales sur le bétail que celles qui sont appliquées aux bergers brigasques - et non de celles qui seraient valables pour des étrangers. Le procès-verbal de ces accords est dressé par les présidents des commissions d'experts, et contresigné par eux, et les trois maires.

Ces accords n'empêchent pas les conflits sur l'usage des pâturages sur le terrain. Les bergers réaldais, leurs 26 troupeaux et leurs 2700 moutons sont verbalisés à dix reprises au cours de l'automne 1950 par le garde communal de la Brigue parce qu'ils dépassent les bornes fixées par les accords d'Ormea, et certains sont cités à comparaître devant le tribunal de Breil-sur-Roya ${ }^{18}$. À nouveau, l'absence d'accord sur des instruments de saisie de ces territoires est au principe du conflit :

"L'article $1^{e r}$ [des accords d'Ormea] ne désignant les pâturages que par leurs noms (Sanson, Polignaga, Colle Ardente) les bergers réaldais en profitèrent pour étendre considérablement la zone qui à leur avis était couverte par ces dénominations. Les Brigasques protestèrent avec violence ${ }^{19}$."

Les présidents des commissions italienne et française tentent une nouvelle fois d'imposer leurs outils administratifs pour régler le conflit en convoquant une autre réunion en mai 1951 afin de fixer les limites des pâturages sur la «carte planimétrique ». Mais au cours des mois suivants les Réaldais continuent à transgresser ces limites, rapporte le pré-

18. Ministère des Affaires étrangères français (par la suite MAE F), 193QO 244. 19. Note du président de la commission des experts civils françaises, J. Brunschwig-Bordier, à M. O'Connor, 8 mai 1952. MAE F, 139QO245. 
sident de la commission française ${ }^{20}$, si bien qu'en avril 1952, le maire de la Brigue dénonce les accords d'Ormea, et dépose plainte devant la justice italienne pour "pâturage abusif (pascolo abusivo) » contre huit bergers réaldais.

Les deux années d'application des accords d'Ormea marquent donc l'incapacité des outils administratifs à saisir et encadrer les usages locaux : les pâturages concernés n'ont jusqu'alors jamais été tracés sur une carte et leurs limites n'ont vraisemblablement jamais fonctionné comme des lignes à ne pas dépasser. L'intensification du conflit entre les bergers réaldais et la mairie de la Brigue conduit à multiplier les acteurs locaux qui y interviennent, dans son côté répressif notamment : garde communal, tribunaux français et italien.

\section{Multiplication Des ACTEURS POLITIQUES ET MONTÉE EN TECHNICITÉ DE LA SAISIE DE L'ESPACE}

Les acteurs locaux ne sont pas les seuls à tenir en échec des outils administratifs de règlement du désaccord relatif aux pâturages de Realdo. Presqu'en même temps que la signature des accords d'Ormea, en juin 1950, le ministère des Affaires étrangères français revient sur l'interprétation du traité sur laquelle étaient jusque-là d'accord Wuillaume et De Dominicis, et demande le renvoi de la question devant une Commission de conciliation prévue par l'article 83 du Traité de paix en cas de différend sur l'application de l'annexe 14. Autrement dit, les négociations sont reprises en main par les ministères, des Affaires étrangères essentiellement. À partir de ce moment, de fait, coïncident la commission de conciliation «politique », et les commissions « administratives » des experts civils, la première ayant la préséance. L'échelon gouvernemental n'était certes pas absent des épisodes précédents : les présidents des commissions d'experts civils, mais également les préfets, reçoivent des instructions de la part de leurs gouvernements respectifs. Mais ces derniers interviennent désormais plus directement dans le conflit.

20. Idem.

\section{Des autorités nationales plurielles : la fragmentation de « la France » et de « l'Italie »}

Jean-Pierre Le Bourhis (2007) étudie l'installation progressive de « conventions standardisées de description » des risques d'inondation en France. Dans un premier temps, les cartes construites selon les logiques locales, et défendues par les élus locaux, sont « à la fois vraies localement et contradictoires à l'échelle de la rivière » (p. 10). L'action conjointe du préfet et de la DDE pour légitimer les outils cartographiques de l'État permet d'aboutir à une description du danger « conforme au modèle posé par l'État, c'est-à-dire exempte des déformations liées au point de vue local» (p. 15). Dans notre cas, les États disposent certes d'outils préexistants tels que le cadastre et la carte d'état-major. Mais ni « l'Italie » ni «la France » ne peuvent ici être conjuguées au singulier : la politique de chaque État à propos des pâturages de Realdo fait l'objet de préconisations partiellement contradictoires de la part d'acteurs locaux et nationaux en concurrence. On est donc loin d'un consensus, au sein de chaque pays, sur les contours de ces "intérêts nationaux » que tous prétendent défendre.

Le jeu des alternances politiques explique en partie les revirements ministériels, chacun s'opposant à son prédécesseur tout en étant partiellement contraint par ses décisions (Aldrin et al., 2016). Or sous la IV e République, ces successions sont particulièrement nombreuses. Cependant, pour la période qui nous intéresse, le ministère des Affaires étrangères français reste aux mains des démocrateschrétiens du MRP, et en particulier entre celles de deux hommes : Georges Bidault de la Libération à juillet 1950, puis de nouveau à partir de janvier 1953, et Robert Schuman entre juillet 1950 et décembre 1952. Le désaveu par le ministère des Affaires étrangères français de l'accord entre les commissions administratives correspond donc au moment du changement de l'occupant du Quai d'Orsay.

En Italie aussi, le ministère des Affaires étrangères est assez stable; il est de plus aux mains de proches du pouvoir central. Alcide De Gasperi est président du Conseil de décembre 1945 à août 1953; il est également ministre des Affaires étrangères de décembre 1944 à octobre 1946, puis de juil- 
let 1951 à août 1953. Le comte Sforza est ministre des Affaires étrangères du gouvernement Badoglio en 1943, puis de février 1947 à juillet 1951. L'ambassadeur d'Italie à Paris de novembre 1946 à juin 1958, Pietro Quaroni, est également un proche conseiller de De Gasperi.

Les évolutions de la composition des gouvernements ne reflètent qu'en partie les enjeux politiques nationaux. Ainsi, alors que Bidault avait négocié en juillet 1948 un accord avec Quaroni qui revenait sur une partie des cessions territoriales, il échoue à le faire adopter par l'Assemblée nationale française, car il est rejeté à l'unanimité par sa commission des Affaires étrangères le 29 décembre 1948. Le point d'achoppement concerne alors la frontière avec la Savoie, qui donne lieu à d'intenses mobilisations locales soutenues par De Gaulle et le PCF et qui isolent le MRP et Bidault, accusés d'une tendresse coupable envers l'Italie en raison notamment de leur catholicisme.

Ces positions politiques ne sont pas les seules lignes de division de «l'État français » : la stratégie relative à la frontière franco-italienne fait aussi l'objet d'un désaccord entre ministères, et oppose en particulier les militaires aux diplomates. Les archives diplomatiques explicitent l'existence de deux positions : l'État-major veut éviter toute possibilité d'attaque par l'est, et souhaite donc maîtriser les crêtes, position justifiée par le rappel incessant du " coup de poignard dans le dos », en référence à l'attaque italienne des Alpes de juin 1940. Les Affaires étrangères sont davantage, comme G. Bidault, partisanes d'une ligne modérée qui donne la priorité aux bonnes relations franco-italiennes au principe de la négociation d'une union douanière, d'accords de main d'œuvre, et de la CECA.

Les archives italiennes n'indiquent pas un tel désaccord entre les Affaires étrangères et l'Étatmajor. Quaroni souligne en revanche « les efforts du Quai d'Orsay sur les autres ministères techniques, peu maniables dans tous les pays ${ }^{21} »$. Et en effet les archives du ministère des Affaires étrangères italien portent la marque de la lassitude des diplomates et de leur mépris à l'égard des questions « techniques » prises en charge par la Commission des experts civils. En réponse, la direction des Affaires politiques du ministère recommande à l'ambas-

21. Ministère des Affaires étrangères italien (par la suite MAE I), AP 45-60, 13. Lettre du 23 octobre 1952. sadeur de privilégier, à l'avenir, «les conversations entre fonctionnaires des deux ministères [des Affaires étrangères] et des ambassades, c'est-à-dire à l'exclusion des "experts" des différentes administrations ${ }^{22}$ ". Ce conflit frontalier fait donc l'objet à la fois de désaccord entre deux ministères « régaliens ", l'armée et les affaires étrangères, et de luttes de territoire entre ces ministères qui se définissent comme "politiques » à l'égard des ministères « techniques».

Ces lignes de clivage, qui traversent les autorités gouvernementales, modèlent également les relations entre les acteurs politiques locaux et nationaux. Au cours de l'été 1949, l'État-major italien adresse d'abord à De Dominicis puis au «bureau frontière »(ufficio confine) de la Présidence du Conseil ses protestations relatives à la teneur des négociations locales ${ }^{23}$. Il estime que le travail de la commission militaire de délimitation de la frontière ne doit pas être remis en cause : «Des problèmes d'ordre économique local ne devraient pas revêtir une importance telle qu'on y sacrifiât l'intérêt militaire. " Concernant les pâturages de Realdo, il estime que ceux-ci doivent effectivement être obtenus par l'Italie pour des raisons économiques; mais que la proposition française de les échanger contre des terres anciennement françaises passées en Italie doit être refusée car elle « camoufle sous un habillage économique des intérêts exclusivement militaires ». L'ensemble des lignes de clivages internes aux États est ainsi décliné dans cette lettre : les ministères régaliens défendant les «intérêts supérieurs » (ici militaires) du pays contre des problèmes « techniques », économiques et en l'occurrence locaux. On perçoit également un assez net soupçon quant aux compétences stratégiques des acteurs administratifs locaux, incapables de distinguer des intérêts militaires « déguisés ». De part et d'autre de la frontière, les directions militaires sont les acteurs nationaux les plus intransigeantes par rapport à son tracé, qu'ils ont établi sur le terrain.

Dans les interactions entre acteurs nationaux et locaux, en revanche, ces derniers sont souvent les plus intraitables. En France, en février 1950, Roger Wuillaume écrit au ministre des Affaires étrangères en regrettant la position « inopportune, maladroite et assez peu élégante » de la mairie de la Brigue à l'égard des bergers réaldais; mais « la

22. Ibidem.

23. CEC, B109 
législation française ne permet pas aux autorités de tutelle de s'immiscer dans la gestion des patrimoines communaux ». Il propose que les ministères de l'Intérieur et des Affaires étrangères demandent au préfet des Alpes-Maritimes « de mettre en jeu, non pas son autorité, mais son influence » sur le maire de la Brigue. À sa suite, une note du service juridique du ministère des Affaires étrangères confirme qu'« aucune clause du traité n'oblige la commune de la Brigue à laisser les habitants de Realdo [...] utiliser leurs anciens pâturages désormais en France ». $\mathrm{Si}$ « le gouvernement français, pour faciliter les rapports de bon voisinage avec l'Italie, souhaite que la municipalité de la Brigue accepte de donner cette autorisation », le préfet peut «l'y inviter » mais ni l'y obliger ni se substituer à elle ${ }^{24}$. C'est sans doute une raison importante du recours, demandé par le ministère des Affaires étrangères français, à la Commission internationale de conciliation : sa décision s'imposera pour sa part aux acteurs politiques locaux.

\section{«L'expertise neutre» pour outrepasser les acteurs politiques locaux}

Ce recours à la commission internationale constitue ainsi un moyen pour s'éloigner des logiques politiques locales qui vont à l'encontre des règlements du conflit imaginés jusque-là. Il s'en éloigne au sens littéral puisque les négociations se déplacent et sont menées par des acteurs sans attache ou expérience locale. Le recours au texte du traité et à une expertise géométrique peuvent également être lus comme des tentatives pour faire prévaloir des logiques «techniques" sur les arguments politiques locaux.

La commission de conciliation franco-italienne est officiellement saisie en vertu d'un échange de lettres du 27 septembre 1951. Elle est composée d'un conseiller d'État représentant la France, d'un président de section honoraire du Conseil d'État représentant l'Italie, et d'un crésident honoraire du tribunal fédéral suisse, tiers-membre choisi d'un commun accord par les couvernements français et italien. On ne dispose malheureusement pas de document d'archive permettant d'éclairer les raisons du choix d'un juge suisse comme tiers-membre. S'agissant de questions frontalières alpines, la neu-

24. Note du 16 février 1950. MAE F, 193QO243. tralité de la Suisse peut sembler étonnante, et d'autant plus celle d'un juge de Morcote, situé dans le Tessin italophone, plus encore sur le lac de Lugano, c'est-à-dire sur la frontière italo-suisse, elle-même objet de nombreux différends. «L'expert neutre » désigné par la commission pour traiter des pâturages de Realdo, le géomètre Renato Solari, vient lui aussi du Tessin, dont il dirige le Bureau des améliorations foncières. Il a travaillé en collaboration avec les experts désignés par les parties : un inspecteur forestier pour l'Italie, un ingénieur des Eaux et Forêts pour la France. Le recours à la commission de conciliation n'écarte pas radicalement les acteurs locaux du processus : à nouveau, la référence faite par le traité aux «besoins des populations » implique de les consulter. "L'expert neutre a entendu les représentants de la commune de la Brigue, les bergers et le curé de Realdo et s'est rendu sur les lieux ${ }^{25}$. »

Plus encore, l'expertise technique n'est pas si facile à imposer en tant que savoir neutre (Delmas 2011). Cette fois, ce sont les représentants des gouvernements qui expriment leur désaccord :

«Ce que les Agents des deux Gouvernements critiquent, en se prévalant de l'opinion de leurs experts, ce sont les appréciations de l'expert neutre sur les données à mettre à la base du calcul [...]. Chaque partie les trouve trop optimistes ou trop pessimistes, selon son intérêt. Il s'agit de questions exclusivement techniques, dans lesquelles la Commission ne peut que s'en tenir à l'avis de l'expert neutre désigné. Les réponses données verbalement par M. Solari, lors de la session de Bordighera, aux objections formulées à cet égard par les deux parties, ont confirmé la Commission dans la conviction que l'expert neutre a jugé la situation objectivement avec science et conscience, en s'abstenant de prévisions teintées de trop d'optimisme ou de trop de pessimisme ${ }^{26}$. "

En réponse, le tiers-membre, qui rédige manifestement le compte-rendu, multiplie les appels à la science et à la technicité : « des questions exclusivement techniques », « objectivement avec science et conscience ", etc. Pourtant, le rapport Solari, dont les conclusions sont adoptées à la seconde version, est loin de mobiliser des arguments uniquement techniques :

25. Procès-verbal de la réunion de San Remo, 1952. CEC, B107. 26. Ibidem. 
« Ces conclusions dépassent peut-être le cadre du problème qu'on nous avait posé. Mais la visite sur place nous a persuadés que les populations montagnardes des petits hameaux de Brigue méritent d'être aidées à améliorer leurs conditions de vie; ceci surtout pour les quatre villages du versant italien.

« En Suisse, en France, en Italie (loi Fanfani) on poursuit une politique d'aide aux populations montagnardes qui doivent être considérées comme une des sources les meilleures de la force des nations. Partout on assiste au phénomène malheureusement de l'abandon des montagnes et partout on cherche à le freiner.

"Le déplacement de la frontière, conséquence d'une guerre malheureuse, ne devrait pas rendre plus difficiles les conditions d'existence déjà dures des habitants de Morignolo ou de Piaggia, Upega, Carnino et Realdo, villages qui dans les derniers 50 ans ont déjà vu leur population diminuer de la moitié.

"Surtout le sort des Réaldais nous préoccupe et la Commission de Conciliation pourra se faire un mérite si, dans l'esprit du Traité, elle trouvera pour ce cas une solution juste et équitable ${ }^{27}$. »

Ces arguments sont repris dans la décision arbitrale finale qui donne raison aux bergers réaldais en vertu du caractère exceptionnel de ces territoires montagnards : "Le Gouvernement français reconnaît que la notion de services communaux doit être ici entendue dans un sens large; il s'agit de petites communes de montagne, pour lesquelles la possession d'un pâturage, d'une forêt, d'un droit de pacage ou d'affouage constituent, le plus généralement, des besoins impérieux et sont d'intérêt public. » Finalement, le recours à une expertise géométrique n'entraine pas une transformation radicale des arguments échangés en termes techniques.

Mais ce n'est pas le seul intérêt du passage par l'échelon international : les décisions de la commission de conciliation s'imposent donc, en droit, aux élus locaux de la Brigue qui y sont pourtant opposés, quand ce n'était le cas ni de celles des commissions administratives ni même de celles du gouvernement français, s'agissant du patrimoine de la commune.

Une marge de manœuvre continue cependant d'appartenir aux acteurs locaux puisque la mise

27. On peut noter dans ce rapport les tournures de phrase italiennes conservées en français (« les derniers 50 ans », « si elle trouvera»). en œuvre de l'aboutissement final de ce processus leur revient : en effet, le bornage des concessions réaldaises en territoire brigasque est à la charge des deux communes concernées, la Brigue et Triora. Lors de sa séance du 3 avril 1955, le Conseil municipal de la Brigue décide de surseoir aux dépenses d'un bornage, dans l'hypothèse où l'on pourrait dans un avenir proche revenir sur cette décision ou trouver une solution au « goût de la Brigue », et menace de démissionner en entier en signe de protestation si on l'y oblige. "Depuis l'administration centrale a insisté [...]; à la Brigue on semble vouloir gagner $d u$ temps ${ }^{28}$ » notent les Renseignements généraux français fin avril, alors que la saison d'usage de ces pâturages approche.

Cet épisode conflictuel relatif aux pâturages de Realdo dessine une situation paradoxale en termes d'appropriation institutionnelle du territoire. D'un côté, les clivages entre les acteurs locaux s'expriment en termes nationalistes : à travers le cas de ces pâturages, chacun défend l'appartenance de cette haute-vallée montagnarde à l'Italie ou à la France. Ce nationalisme des acteurs politiques qui prétendent représenter les populations locales est aussi un moyen pour tenter de convoquer les ressources étatiques à leur service. Certains dispositifs politico-juridiques, qui renvoient à la territorialisation du pouvoir, vont dans ce sens : le traité prévoit des commissions administratives et internationales pour assurer le respect des besoins des populations locales, les rectifications de frontières doivent passer par le Parlement, etc. De l'autre côté, ces territoires occupent des positions périphériques et les outils administratifs des États concernés y sont en réalité peu installés. C'est pourquoi les dispositifs administratifs internationaux prévus pour régler les conflits locaux sont « débordés » par les acteurs locaux dès lors qu'ils ne les satisfont pas.

De ce point de vue, ces territoires évoquent la Zomia étudiée par James Scott (2013), zone montagnarde frontalière dont l'État est absent et même activement repoussé. On a vu en effet que les catégorisations indigènes de l'espace, en l'occurrence des pâturages, fonctionnent de façon assez autonome par rapport aux instruments cadastraux et cartographiques. Mais si l'on veut, en suivant Scott ou encore Sahlins (1996), souligner les capacités

28. AD06, 618W23. 
d'action des populations locales, il serait sans doute plus juste en l'occurrence d'insister sur leur capacité à mobiliser les ressources étatiques pour tenter de prendre l'avantage dans les conflits locaux, en profitant de cette situation frontalière spécifique qui, dans le contexte de l'après-seconde-guerre mondiale, fait de la volonté populaire le principal critère d'appartenance nationale.

Ce cas invite ainsi à examiner les significations prises par les affirmations nationalistes selon leur contexte social et politique. Ici, si l'ensemble des acteurs étudiés fait référence aux intérêts de «son » pays, ceux-ci désignent en réalité des choses différentes. Pour les acteurs locaux, l'intérêt national est celui de «leur» partie de la vallée de la Roya. Pour les deux gouvernements, le cas des pâturages de Realdo constitue davantage un accroc minime dans les relations bilatérales intenses qu'ils développent après-guerre. Pour l'Italie, l'intérêt national prend une dimension encore plus vaste géographiquement si l'on garde à l'esprit que la Roya n'est pas le seul «territoire cédé » par l'Italie : sans même parler de ses colonies, ceux-ci se situent pour l'essentiel sur sa frontière orientale. Or l'ensemble de ces territoires perdus par l'Italie est régi par les mêmes dispositions du traité, et les interprétations données au traité ici seront susceptibles d'être reprises là-bas : les diplomates italiens pensent aussi à la Yougoslavie quand ils négocient dans la Roya. Ainsi, à la diversité des acteurs engagés dans ce conflit territorial répond une multiplicité de rapports au territoire et de formulation d'intérêts « nationaux ».

\section{Bibliographie}

Aldrin P., Bargel L., Bué N. et Pina C. (dir.), 2016. Sociologie des alternances au pouvoir, Vulaines-sur-Seine, le Croquant, $412 \mathrm{p}$.

Amilhat Szary A.L. et Giraut F. (dir.), 2015. Borderities and the politics of contemporary mobile borders, Baginstoke, Palgrave Macmillan, 308 p.
Assier-ANdRIEu L. (dir.), 1990. Une France coutumière: enquête sur les « usages locaux » et leur codification (XIXe-XXe siècles), Paris, CNRS, 207 p.

BARgel L., 2016. Les « originaires » en politique. Migration, attachement local et mobilisations électorales de montagnards, Politix, nº 113, p. 171-199.

Bourdon E., 2011. Le voyage et la découverte des Alpes. Histoire de la construction d'un savoir 1492-1713, Paris, PUPS, $120 \mathrm{p}$.

Couttenier C., Gastaud B., 2007. Aimable Gastaud, 19001974: l'âme du rattachement de Tende et La Brigue à la France en 1947, Nice, Serre, 496 p.

Delmas C., 2011 . Sociologie politique de l'expertise, Paris, La Découverte, $128 \mathrm{p}$.

Dormagen J.-Y., 2008. Logiques du fascisme: L'État totalitaire en Italie, Paris, Fayard, 460 p.

Dumont M., 2011, «Aux origines d'une géopolitique de l'action spatiale : Michel Foucault dans les géographies françaises », L'Espace Politique. Revue en ligne de géographie politique et de géopolitique, 2011, nº 12 .

Harley J. B., 2010. The New Nature of Maps: Essays in the History of Cartography, Baltimore, Johns Hopkins University Press, $360 \mathrm{p}$.

Hoibian O., 2008. L'invention de l'alpinisme: la montagne et l'affirmation de la bourgeoisie cultivée, 1786-1914, Paris, Belin, 380 p.

Kain R.J.P., Baigent E., 1992. The Cadastral Map in the Service of the State: A History of Property Mapping, Chicago, University of Chicago Press, $460 \mathrm{p}$.

Le Bourhis J.-P., 2007. Du savoir cartographique au pouvoir bureaucratique. Les cartes des zones inondables dans la politique des risques (1970-2000), Genèses, vol. 68, n³ 3, p. $75-96$.

Radcliffe S., 2001. Imagining the State as a Space. Territoriality and the Formation of the State in Ecuador, in States of imagination. Ethnographic explorations of the postcolonial state, Durham, Duke University Press, p. 123-145.

Sahlins P., 1996 [1989]. Frontières et identités nationales: la France et l'Espagne dans les Pyrénées depuis le XVII siècle, Paris, Belin, 416 p.

Sсотт J. C., 2013 [2009]. Zomia ou L'art de ne pas être gouverné, Paris, Seuil, 529 p.

Weber M., 1995 [1921], Économie et société, Paris, Pocket, $410+424 \mathrm{p}$.

Wood D., 1992. The Power of Maps, New York, Guilford Press, $248 \mathrm{p}$.

Wood D., 2010. Rethinking the Power of Maps, New York, Guilford Press, $353 \mathrm{p}$. 